Research Paper

\title{
High expression of PDE4D correlates with poor prognosis and clinical progression in pancreaticductal adenocarcinoma
}

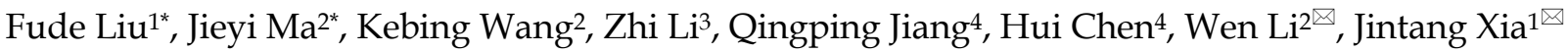 \\ 1. Department of General Surgery, The Third Affiliated Hospital, Guangzhou Medical University, Guangzhou, Guangdong 510150, China \\ 2. Laboratory of General Surgery, The First Affiliated Hospital, Sun Yat-sen University, Guangzhou, Guangdong 510080, China \\ 3. Department of Pathology, The First Affiliated Hospital, Sun Yat-sen University, Guangzhou, Guangdong 510080, China \\ 4. Department of Pathology, The Third Affiliated Hospital, Guangzhou Medical University, Guangzhou, Guangdong 510150, China \\ *These authors contributed equally to this work
}

$\triangle$ Corresponding authors: Jintang Xia, Department of General Surgery, The third Affiliated Hospital, Guangzhou Medical University, No.63 Duobao Road, Guangzhou, Guangdong 510150, China. Phone: +86-15800033336; Fax: +86(20)-81292949; Email: 13xia@163.com. Wen Li, Laboratory of General Surgery, The First Affiliated Hospital, Sun Yat-sen University, No.58 Zhongshan II Road Guangzhou, Guangdong 510080, China. Phone: +86-13728048336; Fax: +86(20)-87755766; Email: liwen@mail.sysu.edu.cn

(1) The author(s). This is an open access article distributed under the terms of the Creative Commons Attribution License (https://creativecommons.org/licenses/by/4.0/). See http://ivyspring.com/terms for full terms and conditions.

Received: 2019.04.02; Accepted: 2019.08.26; Published: 2019.10.17

\begin{abstract}
Background: Phosphodiesterase 4D (PDE4D) has recently been reported as an oncogene in various types of human cancers. However, the expression and significance of PDE4D in pancreatic ductal adenocarcinoma (PDAC) have not been elucidated.

Methods: Immunohistochemistry (IHC) was used to examine the expression of PDE4D in 104 clinicopathologically characterized PDAC cases. PDE4D expression in paired tumor tissues and adjacent noncancerous tissues were detected by western blotting and real time qRT-PCR. The correlation of PDE4D expression levels with clinicopathological features and prognosis in patients were analyzed by univariate and multivariate methods. Effect of PDE4D on pancreatic cancer cells was detected by cell migration and invasion assays.

Results: We found that PDE4D was significantly up-regulated in PDAC tumor tissues compared to those paired adjacent noncancerous tissues at both protein and mRNA levels. High level of PDE4D was significantly associated with clinical stage $(P=0.004)$, T classification $(P=0.003)$, lymph node metastasis $(P=0.022)$ and liver metastasis $(P=0.038)$. Patients with higher levels of PDE4D had shorter overall survival time contrast with those with lower PDE4D expression $(P=0.002)$. Multivariate analysis indicated that PDE4D may be an independent prognostic factor for PDAC. PDE4D depletion significantly suppressed $\beta$-catenin and Snail expression as well as the migration and invasion abilities of pancreatic cancer cells.

Conclusions: Our study reveals that PDE4D up-regulated in PDAC was closely associated with poor prognosis of PDAC patients and multiple aggressive clinicopathological characteristics. PDE4D could be a useful prognostic biomarker and therapeutic target for PDAC.
\end{abstract}

Key words: phosphodiesterase $4 \mathrm{D}$, pancreatic ductal adenocarcinoma, prognosis, $\beta$-catenin

\section{Introduction}

Pancreatic ductal adenocarcinoma (PDAC) is known as one of the most devastating cancer with an extremely poor prognosis. According to the report of GLOBOCAN 2012, there were 65,727 Chinese diagnosed with PDAC, among which 63,662 cases died, accounting for $96.86 \%$ of the newly diagnosed PDAC cases in China during 2012 [1]. PDAC is the fourth leading cause of cancer-related death in the 
United States and the seventh in China with a median survival of 3-6 months and a 5-year survival rate of less than 5\% [2-4]. Despite decades of effort to this disease, the survival rate remains no apparent improvement [5]. So far, the only curative treatment available for PDAC is surgical resection. Unfortunately the vast majority of patients are not diagnosed until late stage in their disease, whereas less than $20 \%$ of patients are surgically respectable at the time of diagnosis [6, 7]. Strong local invasion and early distant metastasis are the main causes for the worse prognosis of PDAC $[8,9]$.

Phosphodiesterases (PDEs) belong to metalloproteinase, which specially degrade the secondary messengers cAMP and cGMP [10, 11]. According to their subcellular distributions, amino acid sequences and substrate-specificity, PDEs are grouped into 11 families (PDE1-11). PDE4D (cAMP specific $3^{\prime}, 5^{\prime}$-cyclic phosphodiesterase $4 \mathrm{D}$ ) belongs to PDE4 families which contains at least 9 isoforms and all the 9 isoforms have the catalytic domain of phosphodiesterase in their carboxyl terminus. PDE4D plays a very important role in regulating cAMP hydrolyzation but does not affect cGMP $[12,13]$. More recently, PDE4D has been identified as a novel tumor-promoting molecular which represents a unique targetable enzyme in various human cancers, such as lung, prostate, melanoma, ovarian, endometrial, colorectal cancer and gastric cancers [14-17]. However, the PDE4D expression and prognostic significance in PDAC has not been reported up to now.

In the present study, we found that the expression of PDE4D was increased in the PDAC samples, compared to their adjacent non-tumor tissues. High expression of PDE4D was correlated with poor prognosis and clinical progression in patients of PDAC. Furthermore, in vitro experiments indicated that PDE4D may promote migration and invasion abilities of PDAC cells through $\beta$-catenin and Snail.

\section{Material and Methods}

\section{Patients and Tissue Specimens}

The tissues, which were archived and formalin-fixed paraffin-embedded, were obtained from104 patients with diagnosis of PDAC who had undergone surgical resection or biopsy from September 2003 to March 2011 in the Department of Hepatobiliary Surgery, the First Affiliated Hospital of Sun Yat-sen University, China. There were 67 patients initially received radical resection and 37 patients received palliative operation. Ultrasound and computed tomography scans had been performed on all of the 104 patients prior to their operation. Postoperative chemotherapy was performed to 23 patients with advanced stage of PDAC, whereas no radiotherapy was performed to any of those patients.

To detect the mRNA and protein levels of PDE4D expression, four matched pairs of fresh PDAC tumor and adjacent non-tumor tissue samples, which at less $2 \mathrm{~cm}$ away from the tumor border, were also obtained from the pancreatectomy specimens. By using histopathology analysis with HE staining in frozen sections, all samples had been confirmed that the cancer lesions comprised of more than $70 \%$ cancer cells without necrosis, and adjacent noncancerous tissues did not have tumor cells. The informed consent of all the patients and the permission from Medical Ethical Committee of the First Affiliated Hospital, Sun Yat-sen University had been obtained before the use of clinical specimens in this study.

\section{RNA isolation and $q R T-P C R$}

The total RNA which derived from tumor tissues of PDAC and the matched paired adjacent noncancerous tissues were extracted using the Trizol reagent (Invitrogen; Carlsbad, USA) according to the manufacturer's protocol. Total RNA was handled with RNAase-free DNase in advance, and $2 \mu \mathrm{g}$ RNA from each specimen was used for cDNA synthesis. qRT-PCR was conducted with LightCycler ${ }^{\circledR} 480$ SYBR Green I Master on LightCycler480 instrument (Roche, Switzerland). Primer sequences used in this study are listed as the following: PDE4D, forward: 5'-ACCATTACCATGCTGATGTGGCCT-3' and reverse: 5'-ACACAGCCTCCAAAGCAGGTG -3'; GAPDH, forward: 5'-CTGACTTCAACAGCGAC ACC-3' and reverse: 5'-TGCTGTAGCCAAATTCG TTG-3'. GAPDH expression was used as an internal reference when conducting the data analysis.

\section{Western blotting}

Samples were lysed in protein lysis buffer which was composed of $50 \mathrm{mM}$ Tris (pH 7.5), $100 \mathrm{mM} \mathrm{NaCl}$, $1 \mathrm{mM}$ EDTA, 0.5\% NP40, 0.5\% TritonX-100, $2.5 \mathrm{mM}$ sodium orthovanadate, $10 \mu \mathrm{M}$ protease inhibitor cocktail, and $1 \mathrm{mM}$ phenylmethylsulfonyl fluoride. Extracted protein was separated by $10 \%$ SDS-PAGE, and transferred onto PVDF membranes (Millipore, Bedford, USA). The membranes were blocked in 5\% milk in $1 \times$ TBST for one hour at room temperature, followed by incubation with the respective primary antibodies at $4^{\circ} \mathrm{C}$ overnight. After washed with TBST, the membranes were incubated with horseradish peroxidase conjugated secondary antibodies (1:5000; CST, USA). An enhanced chemiluminescence (ECL) kit (Millipore, Bedford, USA) was used to visualize protein bands on PVDF membranes. Primary 
antibodies used in this study were listed as the following: mouse anti-GAPDH antibody (1:3000, Kangcheng, Shanghai, China), rabbit anti-PDE4D antibody (1:1000, Proteintech, USA), mouse anti- $\beta$-Catenin antibody (1:1000, BD, USA), mouse anti-N-cadherin antibody and rabbit anti-Snail (1:1000, CST, USA).

\section{Immunohistochemistry}

Using an ultrasensitive kit (MXB; Fuzhou, China), immunohistochemical staining for PDE4D was performed on formalin-fixed, paraffin-embedded sections ( $4 \mu \mathrm{m}$ thick), which were dewaxed in xylene, rehydrated in decreasing gradient ethanol, and then rinsed in PBS followed by antigen retrieval at $100^{\circ} \mathrm{C}$ with high-pressure steam treatment in $10 \mathrm{mM}$ citrate buffer $(\mathrm{pH}, 6.0)$. After treating with peroxidase blocking solution to block the endogenous peroxidase activity for $10 \mathrm{~min}$ and normal nonimmunone serum for $10 \mathrm{~min}$ to reduce nonspecific binding, the sections were incubated with rabbit anti-PDE4D antibody at $4^{\circ} \mathrm{C}$ overnight, washed and then incubated with biotin-conjugated second antibody at room temperature for $10 \mathrm{~min}$. Then the sections were sequentially incubated with streptavidin-peroxidase conjugate for $10 \mathrm{~min}$ and developing with 3, 3 '-diaminobenzidine (DAB) as a chromogen substrate. The nuclei were stained with hematoxylin of Mayer.

Evaluation of the immunohistochemical staining was performed by two researchers independently. The PDE4D staining level was based on the proportion of staining area (positive tumor cells) and the intensity of staining. The staining scores were evaluated as follows. Staining intensity: 0 (no staining), 1 (light yellow, weak staining), 2 (yellow brown, moderate staining), 3 (brown color, strong staining). Staining area: 0 (no positive tumor cells), 1 ( $<10 \%$ positive tumor cells), 2 ( $10 \%$ to $35 \%$ positive tumor cells), 3 ( $35 \%$ to $70 \%$ positive tumor cells), 4 (>70\% positive tumor cells). The PDE4D expression levels were determined by the immunoreactivity staining index (SI), which was calculated by the staining intensity and staining area scores and was assigned values of $0,1,2,3,4,6,8,9$, or 12 , as previously described[18, 19]. For statistical analysis, we classified the PDE4D expression levels as two groups according to the SI: high expression level group ( $\mathrm{SI} \geq 6)$ and low expression level group $(\mathrm{SI} \leq 4)$.

\section{Cell migration and invasion assays}

Cell migration and invasion assays were performed to test the effect of PDE4D on pancreatic cancer cells PANC1 and Bxpc3, using cell culture inserts with PET membrane (Corning, USA) and BD BioCoat $^{\mathrm{TM}}$ Matrigel $^{\mathrm{TM}}$ Invasion Chamber $(8 \mu \mathrm{m}$ pore size) (BD Bioscience, USA), respectively. $24 \mathrm{~h}$ after cells were transfected with PDE4D siRNAs (si-PDE4D-1 and si-PDE4D-2) or negative control siRNA (siNC), cell migration and invasion assays were performed according to the manufacturer's protocols and as previously described [20]. Migration assay and invasion assay were carried out at $7 \mathrm{~h}$ and $12 \mathrm{~h}$ for PANC1 cells respectively, while $20 \mathrm{~h}$ and $36 \mathrm{~h}$ for Bxpc3 cells respectively, after cells were suspended in serum free medium and added to the upper chambers of inserts.

\section{Statistical analysis}

Statistical analysis was performed using the SPSS 22.0 statistical software package (SPSS Inc.; Chicago, USA). The correlations between PDE4D expression and clinicopathologic characteristics in PDAC was carried out with Pearson's Chi-square $\left(\chi^{2}\right)$ test, Fisher's exact test and Spearman's rank correlation. The overall survival (OS) was calculated from the diagnosis date to the death date or the last follow-up date if death did not occur. Survival curve was described using the Kaplan-Meier method and compared using log-rank test between the high and low PDE4D expression cases. Univariate and multivariate Cox regression analyses were carried out to analyze the prognostic significance of PDE4D and other clinicopathologic parameters. $P<0.05$ was considered statistically difference.

\section{Results}

\section{Clinicopathologic characteristics of patients with PDAC}

The patients of PDAC contain 65 males and 39 females with age ranging from 27 to 78 years (median age: 61.5 years). The histological differentiation and clinical stages were defined according to the pathological tumor-node-metastasis (pTNM) classification system, based on the American Joint Committee on Cancer (AJCC) [21]. There were 7 cases with well-differentiation, 67 cases with moderate-differentiation and 30 cases with poordifferentiation. Similarly, 10 cases were classified as stage I, 46 cases as stage II, 28 cases as stage III, and 20 cases as stage IV. The correlations of PDE4D with clinicopathologic features in PDAC patients are listed in Table 1.

\section{Expression of PDE4D is up-regulated in PDAC}

We first used Oncomine database to analysis the expression of PDE4D in PDAC [22-25]. These data indicated that PDE4D mRNA was significantly up-regulated in PDAC cohorts (Figure 1A-D). Similar trend was observed in our present study. The protein and mRNA levels of PDE4D were higher in all four 
PDAC lesion tissues (T), compared to their matched adjacent noncancerous tissues $(\mathrm{N})$ as shown in the Figure $1 \mathrm{E}$ and $1 \mathrm{~F}$.

\section{Over expression of PDE4D is correlated with clinicopathologic features of PDAC}

Immunohistochemical staining was performed in 104 cases of PDAC. PDE4D was mainly localized in the cytoplasm of the PDAC tumor cells (Figure 2A). Analysis of the immunohistochemical staining in 104 cases of PDAC, we found that 72 cases $(69.2 \%)$ have strong staining in lesion tissues with $\mathrm{SI} \geq 6$ which was classified as PDE4D high expression level group. The other 32 cases (30.8\%) of PDAC have weak staining in lesion tissues with $\mathrm{SI} \leq 4$ which was classified as PDE4D low expression level group (Table 1).

Table 1. Correlations between PDE4D expression and clinicopathologic features in PDAC

\begin{tabular}{|c|c|c|c|c|}
\hline \multirow{2}{*}{$\begin{array}{l}\text { Clinicopathological } \\
\text { feature }\end{array}$} & \multirow[t]{2}{*}{ Number } & \multicolumn{2}{|c|}{ Expression of PDE4D } & \multirow{2}{*}{$\begin{array}{l}\text { P value } \\
\text { ( } 2 \text { test })\end{array}$} \\
\hline & & Low $(n=32,30.8 \%)$ & $\operatorname{High}(\mathrm{n}=72,69.2 \%)$ & \\
\hline \multicolumn{5}{|l|}{ Age (years) } \\
\hline$\leq 60$ & 45 & 14(31.1\%) & $31(68.9 \%)$ & \multirow{2}{*}{0.947} \\
\hline$>60$ & 59 & $18(30.5 \%)$ & $41(69.5 \%)$ & \\
\hline \multicolumn{5}{|l|}{ Gender } \\
\hline Male & 65 & $23(35.4 \%)$ & $42(64.6 \%)$ & \multirow[t]{2}{*}{0.188} \\
\hline female & 39 & $9(23.1 \%)$ & $30(76.9 \%)$ & \\
\hline \multicolumn{5}{|l|}{ Tumor location } \\
\hline Head & 83 & $28(33.7 \%)$ & $55(66.3 \%)$ & \multirow[t]{2}{*}{0.193} \\
\hline Body/Tail & 21 & $4(19.0 \%)$ & $17(81.0 \%)$ & \\
\hline \multicolumn{5}{|l|}{ Size } \\
\hline$\leq 2 \mathrm{~cm}$ & 15 & $6(40.0 \%)$ & $9(60.0 \%)$ & \multirow[t]{2}{*}{0.402} \\
\hline$>2 \mathrm{~cm}$ & 89 & $26(29.2 \%)$ & $63(70.8 \%)$ & \\
\hline \multicolumn{5}{|c|}{ Clinical stage (pTNM) } \\
\hline I & 10 & $3(30.0 \%)$ & $7(70.0 \%)$ & \multirow[t]{4}{*}{$0.004^{*}$} \\
\hline II & 46 & $10(21.7 \%)$ & $36(78.3 \%)$ & \\
\hline III & 28 & $16(57.1 \%)$ & $12(42.9 \%)$ & \\
\hline IV & 20 & $3(15.0 \%)$ & $17(85.0 \%)$ & \\
\hline \multicolumn{5}{|c|}{ Histological differentiation } \\
\hline Well & 7 & $0(0)$ & $7(100 \%)$ & \multirow[t]{2}{*}{0.161} \\
\hline Moderate/poor & 97 & $32(33.0 \%)$ & $65(67.0 \%)$ & \\
\hline \multicolumn{5}{|l|}{$\mathrm{T}$ classification } \\
\hline T1 & 5 & $2(40.0 \%)$ & $3(60.0 \%)$ & \multirow[t]{4}{*}{$0.003^{*}$} \\
\hline $\mathrm{T} 2$ & 18 & $5(27.8 \%)$ & $13(72.2 \%)$ & \\
\hline T3 & 44 & $6(13.6 \%)$ & $38(86.4 \%)$ & \\
\hline $\mathrm{T} 4$ & 37 & $19(51.4 \%)$ & $18(48.6 \%)$ & \\
\hline \multicolumn{5}{|l|}{$\mathrm{N}$ classification } \\
\hline Absent & 54 & $22(40.7 \%)$ & $32(59.3 \%)$ & \multirow[t]{2}{*}{$0.022^{*}$} \\
\hline Present & 50 & $10(20.0 \%)$ & $40(80.0 \%)$ & \\
\hline \multicolumn{5}{|l|}{ Liver Metastasis } \\
\hline Absent & 84 & $22(26.2 \%)$ & $62(73.8 \%)$ & \multirow[t]{2}{*}{$0.038^{*}$} \\
\hline present & 20 & $10(50.0 \%)$ & $10(50.0 \%)$ & \\
\hline \multicolumn{5}{|l|}{ Resectability } \\
\hline Radical resection & 67 & $26(38.8 \%)$ & $41(61.2 \%)$ & \multirow[t]{3}{*}{$0.017^{*}$} \\
\hline Palliative resection & 37 & $6(16.2 \%)$ & $31(83.8 \%)$ & \\
\hline \multicolumn{4}{|l|}{ Vital status } & \\
\hline Dead & 93 & $26(28.0 \%)$ & $67(72 \%)$ & \multirow[t]{2}{*}{0.071} \\
\hline Alive & 11 & $6(54.5 \%)$ & $5(45.5 \%)$ & \\
\hline
\end{tabular}

The correlations between the protein expression of PDE4D and clinicopathologic features (including age, gender, clinical stage, differentiation and metastasis) were analyzed with Chi-square $\left(\chi^{2}\right)$ test. The results showed that overexpression of PDE4D was significantly associated with clinical stage $(P=$
$0.004)$, T classification $(P=0.003), \mathrm{N}$ classification $(P=$ $0.022)$, liver metastasis $(P=0.038)$ and tumor respectability $(P=0.017)$. No obvious correlations were observed with age, gender, tumor location, size, histological differentiation and other clinicopathologic features (Table 1).

\section{The prognosis significance of PDE4D expression in PDAC patients}

We examined PDE4D expression levels and the clinical follow-up information in all 104 PDAC patients by Kaplan-Meier analysis and log-rank test. Up to the end of follow-up time, 93 patients were died and 11 patients were still alive. Using univariate and multivariate analyses, the crude and adjusted relative risks of all cause of death in the 104 patients were assessed (Table 2). The median survival times of patients with high PDE4D expression level group was 6.5 months [95\% confidence interval (CI): 7.7-11.4 months], which was significantly shorter than 14.3 months [95\% CI: 11.5-21.2 months] for patients with low PDE4D expression level group $(P=0.002$, log-rank text, Figure 2B). The results indicated that patients with high PDE4D expression levels have a worse prognosis compared to those with low PDE4D expression levels. Furthermore, we also examined the median survival times of the patients with different clinical stages, with or without lymph node and liver metastasis by Kaplan-Meier analyses. Our results showed that patients in PDE4D high expression level group with both early (I-II) and advanced clinical stage (III-IV) had markedly shorter survival times compared with those in PDE4D low expression level group (both $P<0.05$; Figure 3A). Similar results were determined between patients in the following two expression groups, with and without lymph node metastasis (both $P<0.05$; Figure 3B), with and without liver metastases (both $P<0.05$; Figure 3C).

To determine whether PDE4D expression level is an independent prognostic factor, univariate and multivariate analyses were performed in the 104 cases of PDAC. As is shown in Table 2, univariate analysis suggested that PDE4D expression, tumor size, $\mathrm{T}$ classification, $\mathrm{N}$ classification and clinical stage were significant prognostic factors in PDAC. And multivariate analysis indicated that PDE4D expression, $\mathrm{T}$ classification and $\mathrm{N}$ classification were determined as independent prognostic factors for poor overall survival of PDAC patients.

\section{PDE4D regulates pancreatic cancer cells metastasis ability through $\boldsymbol{\beta}$-catenin and Snail}

In order to illuminate the effect of PDE4D on pancreatic cancer cells, cell migration and invasion assays were performed. As showed in Figure 4B and 
4C, down-regulation of PDE4D with PDE4D siRNA significantly inhibited the migration and invasion abilities of pancreatic cancer cells indicating that PDE4D played an important role in pancreatic cancer cell metastasis. Mechanism by which PDE4D affects migration and invasion function of pancreatic cancer cells was further investigated. We found that $\beta$-catenin, Snail and $\mathrm{N}$-cadherin protein levels were

A

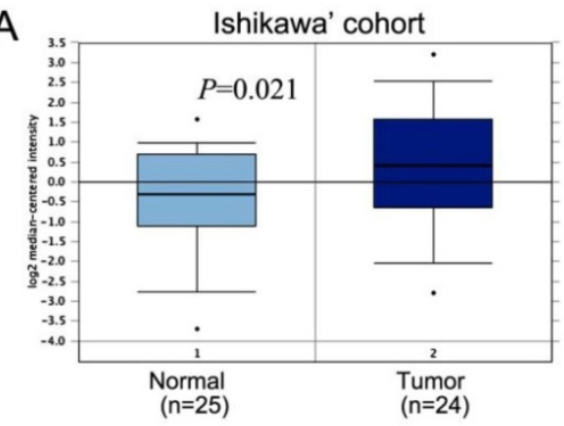

C

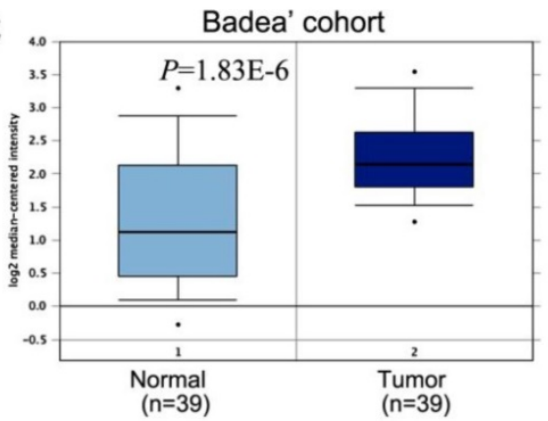

E
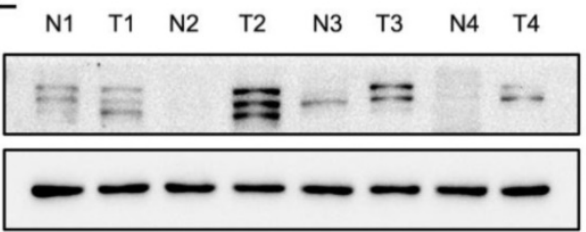

dramatically decreased in PDE4D silenced cells (Figure 4D). $\beta$-catenin is known as activator of the TCF/LEF family, leading to activation of Wnt signaling responsive genes and facilitate metastasis[26]. These data suggest that PDE4D over-expression might be a positive regulator of metastasis in pancreatic cancer cell though $\beta$-catenin/Snail pathway.

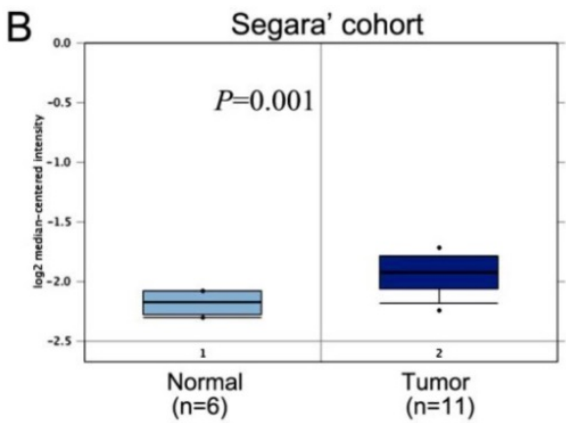

D
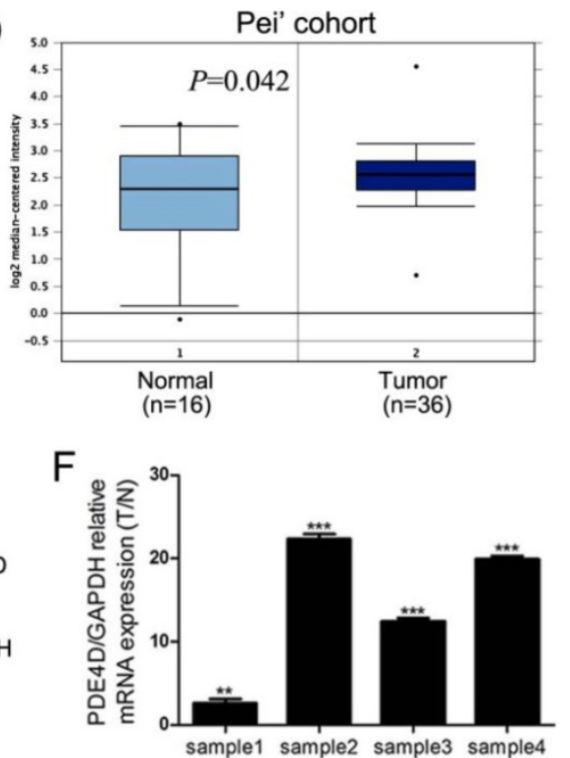

Figure 1. The levels of PDE4D expression is elevated in PDAC. A-D) Oncomine analysis of PDE4D expression in PDAC cohorts. E) Western blot showed protein levels of PDE4D expression in four PDAC tumor tissues (T) and their paired adjacent noncancerous tissues (N). F) qRT-PCR analysis showed the mRNA levels of PDE4D expression in tumor tissues compared to their paired adjacent noncancerous tissues $(\mathrm{T} / \mathrm{N})$. ** $P<0.01$; *** $P<0.001$.

Table 2. Univariate and multivariate analyses of prognostic parameters for survival in patients with pancreatic ductal adenocarcinoma (PDAC)

\begin{tabular}{|c|c|c|c|c|c|c|c|c|}
\hline \multirow[t]{3}{*}{ Prognostic parameter } & \multicolumn{4}{|c|}{ Univariate analysis } & \multicolumn{4}{|c|}{ Multivariate analysis } \\
\hline & \multirow[t]{2}{*}{ RR } & \multicolumn{2}{|l|}{$95 \% \mathrm{Cl}$} & \multirow[t]{2}{*}{$P$ value } & \multirow[t]{2}{*}{ RR } & \multicolumn{2}{|c|}{$95 \% \mathrm{Cl}$} & \multirow[t]{2}{*}{$P$ value } \\
\hline & & Lower & Upper & & & Lower & Upper & \\
\hline Expression of PDE4D & 2.099 & 1.295 & 3.4 & $0.003^{*}$ & 3.272 & 1.84 & 5.817 & $0.000^{*}$ \\
\hline Gender & 1.056 & 0.691 & 1.614 & 0.802 & 0.961 & 0.601 & 1.538 & 0.868 \\
\hline Age & 1.42 & 0.928 & 2.173 & 0.106 & 1.415 & 0.904 & 2.215 & 0.129 \\
\hline Size & 2.055 & 1.112 & 3.795 & $0.021^{*}$ & 1.606 & 0.825 & 3.125 & 0.164 \\
\hline Tumor location & 1.085 & 0.639 & 1.841 & 0.764 & 0.958 & 0.535 & 1.716 & 0.886 \\
\hline Pathologic differentiation & 1.291 & 0.823 & 2.026 & 0.267 & 1.188 & 0.723 & 1.951 & 0.497 \\
\hline $\mathrm{T}$ classification & 1.453 & 1.119 & 1.887 & $0.005^{*}$ & 1.715 & 1.227 & 2.395 & $0.002^{*}$ \\
\hline $\mathrm{N}$ classification & 2.015 & 1.318 & 3.08 & $0.001^{*}$ & 1.717 & 1.092 & 2.702 & $0.019^{*}$ \\
\hline Liver metastasis & 1.385 & 0.84 & 2.283 & 0.201 & 1.813 & 0.962 & 3.419 & 0.066 \\
\hline Clinical stage & 1.358 & 1.09 & 1.691 & $0.006^{*}$ & 1.221 & 0.925 & 1.61 & 0.158 \\
\hline
\end{tabular}

Univariate analysis and multivariate analyses were analyzed by Cox regression.

*Statistically significant; RR: Relative Risk; 95\% CI: 95\% confidence interval. 
A

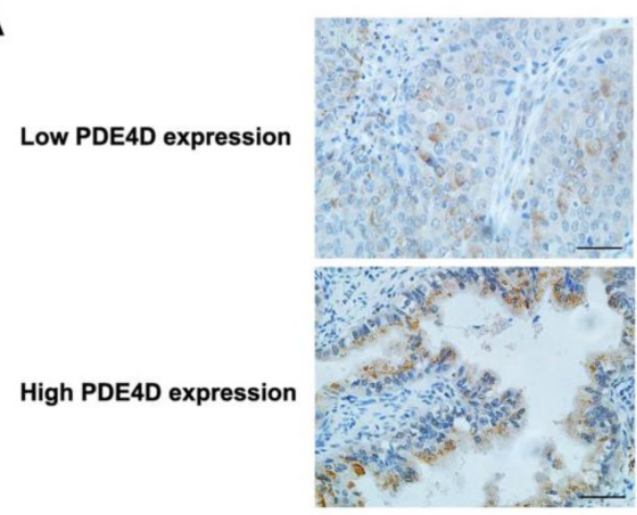

B

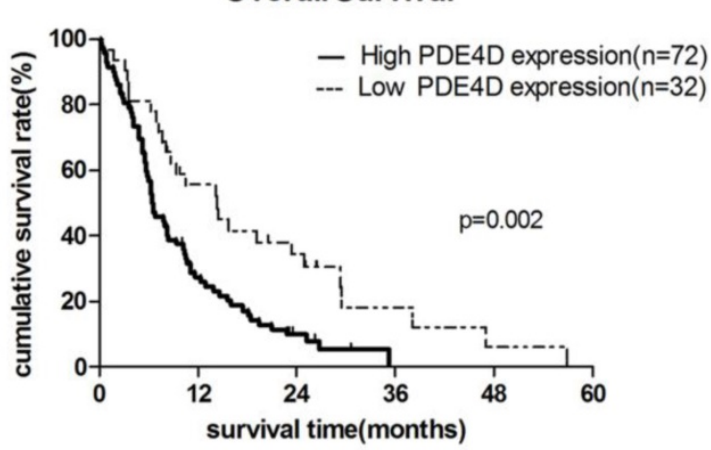

Figure 2. The expression of PDE4D is associated with overall survival in patients with PDAC. A) Immunohistochemical staining showed the low and high expression levels of PDE4D in PDAC tumor tissues, Scale bar: $100 \mu \mathrm{m}$. B) Kaplan-Meier survival curves show the significant statistical differences in survival times between the two groups. $P$-values were given by log-rank test.

A

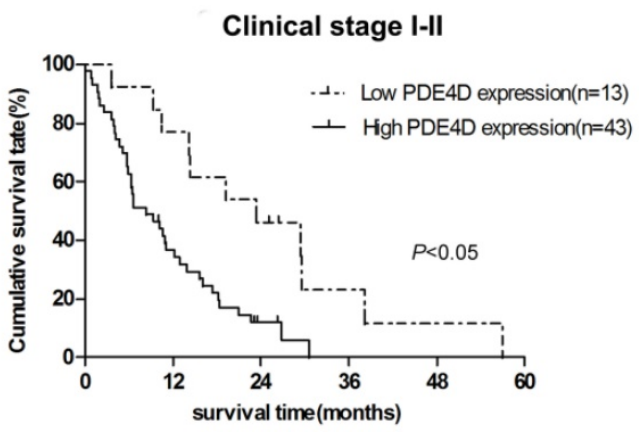

B

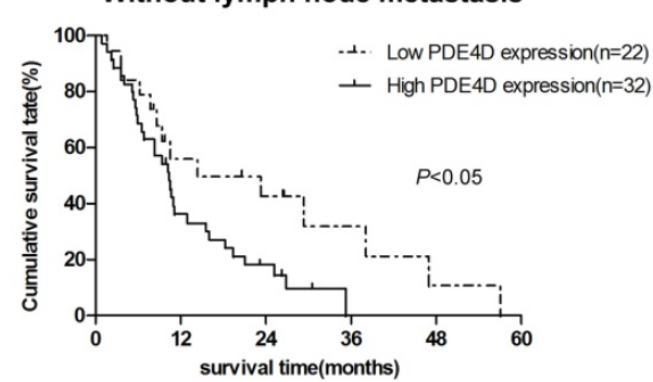

C

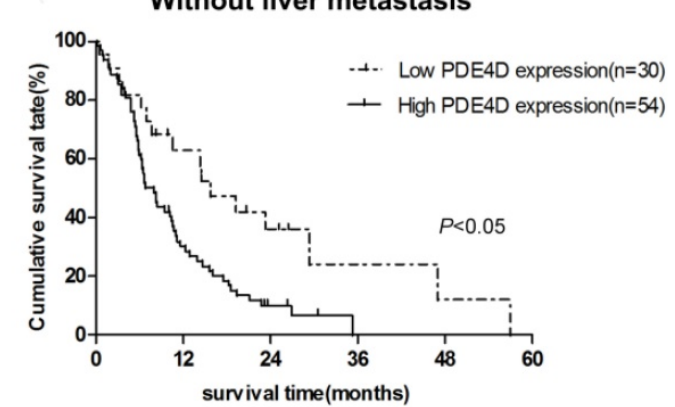

Clinical stage III-IV

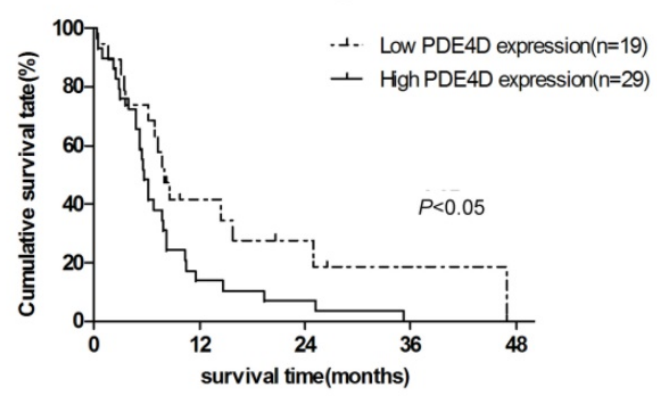

With lymph node metastasis

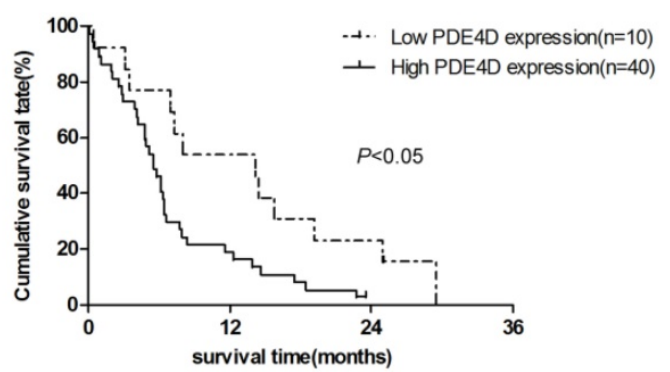

With liver metastasis

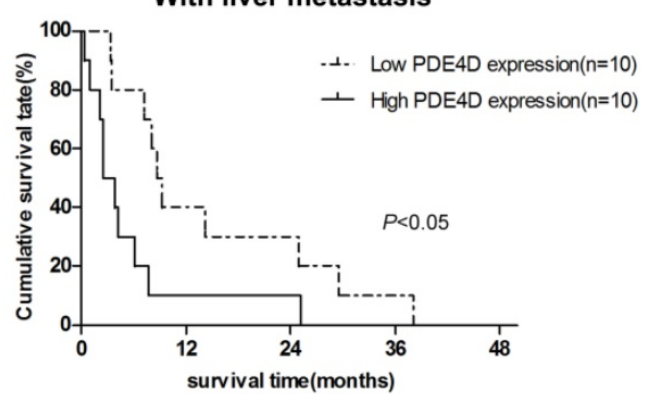

Figure 3. Kaplan-Meier survival curves indicate the significant differences in overall survival between PDAC patients with PDE4D-high and PDE4D-low according to clinical stage (A), lymph node metastasis status (B) and liver metastasis status (C). P-values were given by log-rank test. 
A
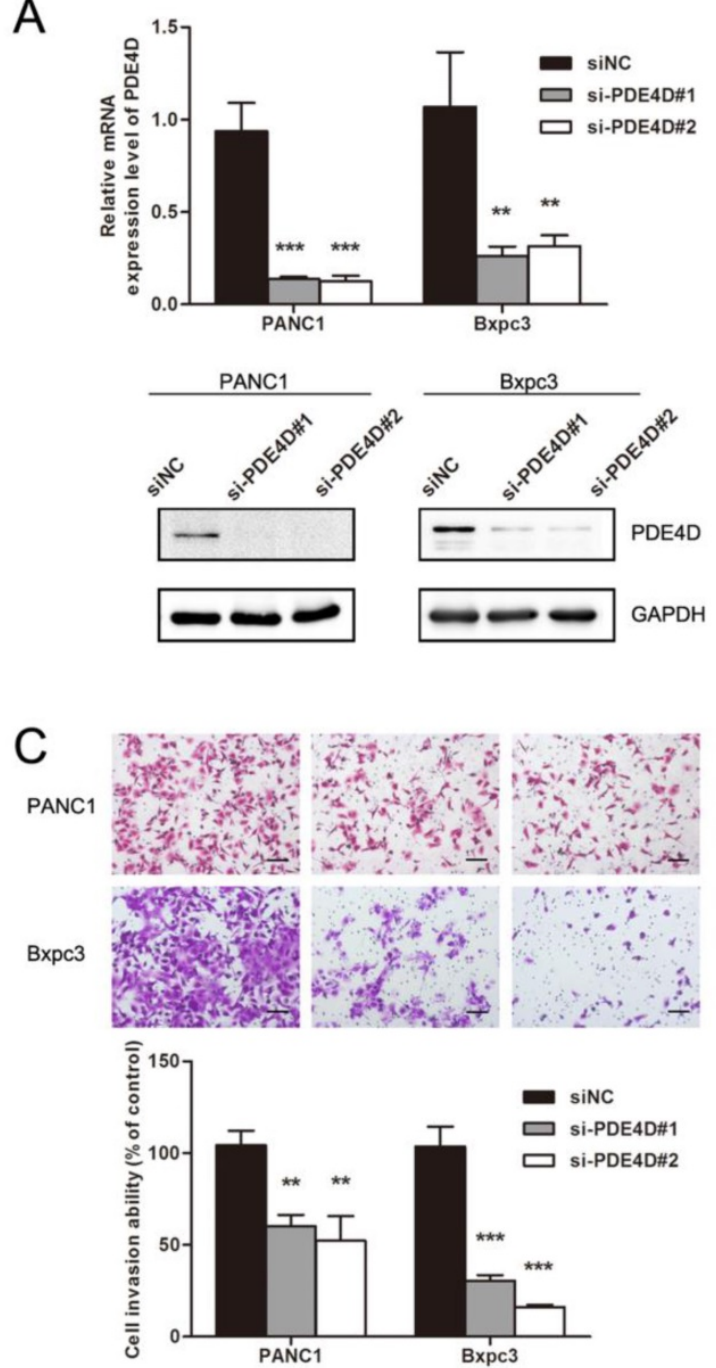

B

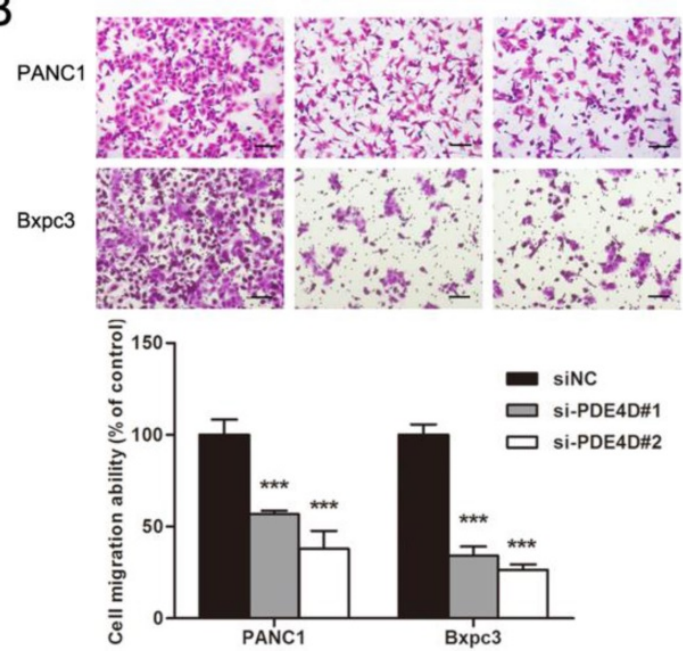

D

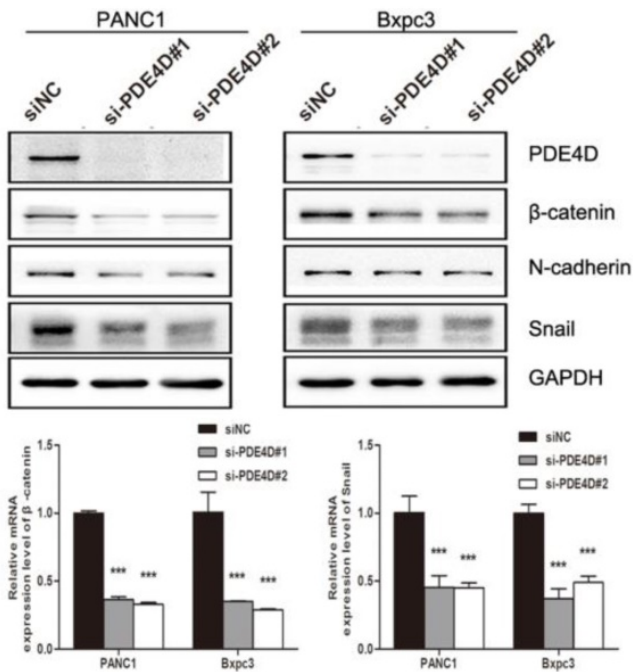

Figure 4. Down-regulation of PDE4D suppressed the metastasis abilities of pancreatic cancer cells. A) PDE4D siRNAs and siNC were transfected into pancreatic cancer cells PANCl and Bxpc3. Western blot and qRT-PCR assay were performed 48h after transfection to examine the effect of siRNAs. B-C) Down-regulation of PDE4D significantly suppressed the migration and invasion abilities of pancreatic cancer cells. Scale bar: $200 \mu \mathrm{m}$. D) Western blotting and qPCR showed that the expression of $\beta$-catenin and Snail were down-regulated coincident with the suppression of PDE4D. Data are presented as mean \pm SD. Statistical analysis was performed using Graph Pad Prism 5 with one-way ANOVA followed by Dunnett's multiple comparison test. $* *: P<0.01$, ***: $P<0.001$.

\section{Discussion}

The poor outcome of PDAC attributes to early and aggressive local invasion, high recurrence rate, and poor sensitive to chemotherapy and radiotherapy $[27,28]$. Negative margin status and absence of lymph node metastases are critical for achieving a better survival rate of this devastating disease $[29,30]$.

cAMP functions as a second messenger in cell signaling transduction and cAMP signaling is regulated mainly by PDE family, especially PDE4D. PDE4D has recently been implicated as an oncogene and is correlated with the growth, proliferation and survival of cancer cells [14-16]. High expression of PDE4D (subtype PDE4D2) significantly enhanced the proliferation of A375 melanoma cells and HGC-27 gastric cancer cells both in vitro and in vivo. Depletion of PDE4D with small interfering RNA (siRNA) caused apoptosis and growth retardation in various types of cancer cells, such as melanoma, breast, ovarian, endometrial, lung, colorectal and gastric cancers[14, 17]. Pharmacologic inhibition of PDE4D using small-molecule inhibitors induce tumor growth inhibition correlated to the $\mathrm{SHH}$ pathway in prostate cancer [31]. Recent study suggested that the mitochondrial-associated apoptosis pathway was activated in shPDE4D-infected cells and the proapoptotic protein BIM was markedly elevated upon PDE4D depletion in both A549 and MB-231 cells [14]. Our previous study found that down-regulation of PDE4D caused BIM-mediated cell growth arrest in colorectal cancer cells [17].

Elevated expression level of PDE4D has been reported to be associated with the proliferation, survival and prognostic in various cancers. However, 
seldom studies have reported the association of PDE4D with migration, invasion and cancer metastasis. In the present work, we found that PDE4D was up-regulated in the tumor tissue of PDAC at both mRNA and protein level compared with non-tumor tissues. For the first time, we demonstrated that overexpression of PDE4D was correlated with clinical stage (pTNM), lymph node metastases and liver metastases in PDAC. Besides, we showed that the group of patients with PDE4D high expression had a markedly shorter survival times compared to the group with PDE4D low expression in PDAC patients. Multivariate analysis of Cox regression indicated that overexpression of PDE4D was an independent factor of poor prognosis. Thus, PDE4D had potential for being used as a prognostic indicator biomarker in patients with PDAC.

Previous study found that PDE4D contributed to TGF- $\beta 1$ stimulated-epithelial mesenchymal transition (EMT) in A549 cells [32]. Recent study showed that PDE4D5 could promote BRAF-mutated melanoma cells invasion by interacting with FAK [33]. Migration suppression was also found in PED4D knockdown prostate cancer cells [16]. Increased levels of cAMP, the PDE4D target, specifically hindered PDAC cell motility through F-actin remodeling [34]. Our in vitro study found that PDE4D could affect migration and invasion function of PDAC cell lines through $\beta$-catenin/Snail pathway.

$\beta$-catenin is a key component of Wnt signaling which is associated with cancer progression and maintains cancer stem cells properties. Studies have confirmed that $\beta$-catenin is critical in cancer cells invasion. Inhibition of AKT/GSK-3 $\beta / \beta$-catenin pathway inhibited cell migration and invasion in hepatocellular carcinoma cells [35]. Via regulating miR-182 and MMP9, $\beta$-catenin also altered cell invasion ability in HCT116 human colorectal carcinoma cells [36]. In the present study, PDE4D silencing attenuated migration and invasion abilities of PDAC cells and decreased $\beta$-catenin level simultaneously. Previous studies have reported that knockdown of PDE4D resulted in EGFR/PI3K/AKT signaling inactivation in nasopharyngeal carcinoma cells [37]. And EGF/AKT could contribute to phosphorylation of $\beta$-catenin and increased its protein level in cancer cells, therefore, enhanced $\beta$-catenin downstream transcriptional activity [38, 39]. Our results indicated that PDE4D could regulate $\beta$-catenin and snail to promote cell invasion in PDAC cells. Whether this process is mediated by EGF/AKT signaling needs further investigation.

In summary, this study demonstrated that high expression of PDE4D was correlated with poor survival in PDAC. PDE4D depletion suppressed migration and invasion of PDAC cells through $\beta$-catenin/Snail pathway. Our study suggests that PDE4D can be used as a novel prognostic biomarker and a potential molecular therapeutic target in patients with PDAC.

\section{Acknowledgements}

We thank Baogang Peng, Lijian Liang (Department of Hepatobiliary Surgery, The First Affiliated Hospital, Sun Yat-sen University) for the help in collection of PDAC tissue samples.

\section{Funding}

This work was supported by grants from the National Natural Science Foundation of China (grant numbers 81672417, 81370368, 81172337), the Science and Technology Foundation of Guangdong province Grant (grant number 2014A020212647), and the Medical Scientific Research Foundation of Guangdong Province (A2018022).

\section{Author Contributions}

W.L. and J.X. conceived, supervised the study and designed experiments; F.L., J.M. and K.W. performed experiments; Z.L., Q.J. and H.C. contributed to collection of fresh tissue samples and analyzed data; F.L. and J.M. wrote the manuscript; W.L. and J.X. made manuscript revisions. All authors have read the final version of the manuscript and are in agreement for publication upon acceptance.

\section{Ethics approval and consent to participate}

The written informed consent of all the patients and the permission from Medical Ethical Committee of the First Affiliated Hospital, Sun Yat-sen University had been obtained before the use of clinical specimens in this study.

\section{Competing Interests}

The authors have declared that no competing interest exists.

\section{References}

1. [Internet] GLOBOCAN 2012. Lyon: IARC. Available online: http://globocan.iarc.fr/.

2. Siegel RL, Miller KD, Jemal A. Cancer statistics, 2015. CA: a cancer journal for clinicians. 2015; 65: 5-29.

3. Chen W, Zheng R, Zeng H, Zhang S, He J. Annual report on status of cancer in China, 2011. Chinese journal of cancer research $=$ Chung-kuo yen cheng yen chiu. 2015; 27: 2-12.

4. Lin QJ, Yang F, Jin C, Fu DL. Current status and progress of pancreatic cancer in China. World journal of gastroenterology. 2015; 21: 7988-8003.

5. Sharma C, Eltawil KM, Renfrew PD, Walsh MJ, Molinari M. Advances in diagnosis, treatment and palliation of pancreatic carcinoma: 1990-2010. World journal of gastroenterology. 2011; 17: 867-97.

6. Ryan DP, Hong TS, Bardeesy N. Pancreatic adenocarcinoma. The New England journal of medicine. 2014; 371: 1039-49.

7. Sutton JM, Abbott DE. Neoadjuvant therapy for pancreas cancer: past lessons and future therapies. World journal of gastroenterology. 2014; 20: 15564-79.

8. Vincent A, Herman J, Schulick R, Hruban RH, Goggins M. Pancreatic cancer. Lancet. 2011; 378: 607-20. 
9. He Y, Zheng R, Li D, Zeng H, Zhang S, Chen W. Pancreatic cancer incidence and mortality patterns in China, 2011. Chinese journal of cancer research = Chung-kuo yen cheng yen chiu. 2015; 27: 29-37.

10. Bender AT, Beavo JA. Cyclic nucleotide phosphodiesterases: molecular regulation to clinical use. Pharmacological reviews. 2006; 58: 488-520.

11. Conti M, Beavo J. Biochemistry and physiology of cyclic nucleotide phosphodiesterases: essential components in cyclic nucleotide signaling. Annual review of biochemistry. 2007; 76: 481-511.

12. Houslay MD. PDE4 cAMP-specific phosphodiesterases. Progress in nucleic acid research and molecular biology. 2001; 69: 249-315.

13. Chandrasekaran A, Toh KY, Low SH, Tay SK, Brenner S, Goh DL. Identification and characterization of novel mouse PDE4D isoforms: molecular cloning, subcellular distribution and detection of isoform-specific intracellular localization signals. Cellular signalling. 2008; 20: 139-53.

14. Lin DC, Xu L, Ding LW, Sharma A, Liu LZ, Yang H, et al. Genomic and functional characterizations of phosphodiesterase subtype $4 \mathrm{D}$ in human cancers. Proceedings of the National Academy of Sciences of the United States of America. 2013; 110: 6109-14.

15. Pullamsetti SS, Banat GA, Schmall A, Szibor M, Pomagruk D, Hanze J, et al. Phosphodiesterase-4 promotes proliferation and angiogenesis of lung cancer by crosstalk with HIF. Oncogene. 2013; 32: 1121-34.

16. Rahrmann EP, Collier LS, Knutson TP, Doyal ME, Kuslak SL, Green LE, et al. Identification of PDE4D as a proliferation promoting factor in prostate cancer using a Sleeping Beauty transposon-based somatic mutagenesis screen. Cancer research. 2009; 69: 4388-97.

17. Cao B, Wang K, Liao JM, Zhou X, Liao P, Zeng SX, et al. Inactivation of oncogenic cAMP-specific phosphodiesterase $4 \mathrm{D}$ by miR-139-5p in response to p53 activation. eLife. 2016; 5.

18. Li W, Yu CP, Xia JT, Zhang L, Weng GX, Zheng HQ, et al. Sphingosine kinase 1 is associated with gastric cancer progression and poor survival of patients. Clinical cancer research : an official journal of the American Association for Cancer Research. 2009; 15: 1393-9.

19. Zhang LJ, Wang KB, Liu LS, Chen LZ, Peng BG, Liang LJ, et al. Overexpression of GOLPH3 is associated with poor prognosis and clinical progression in pancreatic ductal adenocarcinoma. BMC cancer. 2014; 14: 571.

20. Ouyang $\mathrm{M}$, Wang $\mathrm{H}, \mathrm{Ma} \mathrm{J}, \mathrm{Lu} \mathrm{W}$, Li J, Yao C, et al. COP1, the negative regulator of ETV1, influences prognosis in triple-negative breast cancer. BMC cancer. 2015; 15: 132

21. Edge SB, American Joint Committee on Cancer. AJCC cancer staging manual. 7th ed. New York: Springer; 2010

22. Ishikawa M, Yoshida K, Yamashita Y, Ota J, Takada S, Kisanuki H, et al. Experimental trial for diagnosis of pancreatic ductal carcinoma based on gene expression profiles of pancreatic ductal cells. Cancer science. 2005; 96: 387-93.

23. Segara D, Biankin AV, Kench JG, Langusch CC, Dawson AC, Skalicky DA, et al. Expression of HOXB2, a retinoic acid signaling target in pancreatic cancer and pancreatic intraepithelial neoplasia. Clinical cancer research : an official journal of the American Association for Cancer Research. 2005; 11: 3587-96.

24. Badea L, Herlea V, Dima SO, Dumitrascu T, Popescu I. Combined gene expression analysis of whole-tissue and microdissected pancreatic ductal adenocarcinoma identifies genes specifically overexpressed in tumor epithelia. Hepato-gastroenterology. 2008; 55: 2016-27.

25. Pei H, Li L, Fridley BL, Jenkins GD, Kalari KR, Lingle W, et al. FKBP51 affects cancer cell response to chemotherapy by negatively regulating Akt. Cancer cell. 2009; 16: 259-66.

26. Clevers H, Nusse R. Wnt/beta-catenin signaling and disease. Cell. 2012; 149: 1192-205.

27. Singh $P$, Srinivasan R, Wig JD. Major molecular markers in pancreatic ductal adenocarcinoma and their roles in screening, diagnosis, prognosis, and treatment. Pancreas. 2011; 40: 644-52

28. Hsu CC, Herman JM, Corsini MM, Winter JM, Callister MD, Haddock MG, et al. Adjuvant chemoradiation for pancreatic adenocarcinoma: the Johns Hopkins Hospital-Mayo Clinic collaborative study. Annals of surgical oncology. 2010; 17: 981-90.

29. Tempero MA, Malafa MP, Behrman SW, Benson AB, 3rd, Casper ES, Chiorean EG, et al. Pancreatic adenocarcinoma, version 2.2014: featured updates to the NCCN guidelines. Journal of the National Comprehensive Cancer Network : JNCCN. 2014; 12: 1083-93.

30. Shi M, Yu DH, Chen Y, Zhao CY, Zhang J, Liu QH, et al. Expression of fibroblast activation protein in human pancreatic adenocarcinoma and its clinicopathological significance. World journal of gastroenterology. 2012; 18: 840-6.

31. Powers GL, Hammer KD, Domenech M, Frantskevich K, Malinowski RL, Bushman W, et al. Phosphodiesterase 4D inhibitors limit prostate cancer growth potential. Molecular cancer research : MCR. 2015; 13: 149-60.

32. Kolosionek E, Savai R, Ghofrani HA, Weissmann N, Guenther A, Grimminger $\mathrm{F}$, et al. Expression and activity of phosphodiesterase isoforms during epithelial mesenchymal transition: the role of phosphodiesterase 4. Molecular biology of the cell. 2009; 20: 4751-65.

33. Delyon J, Servy A, Laugier F, Andre J, Ortonne N, Battistella M, et al. PDE4D promotes FAK-mediated cell invasion in BRAF-mutated melanoma. Oncogene. 2017; 36: 3252-62.

34. Zimmerman NP, Roy I, Hauser AD, Wilson JM, Williams CL, Dwinell MB. Cyclic AMP regulates the migration and invasion potential of human pancreatic cancer cells. Molecular carcinogenesis. 2015; 54: 203-15.
35. Jian Q, Yang Z, Shu J, Liu X, Zhang J, Li Z. Lectin BS-I inhibits cell migration and invasion via AKT/GSK-3beta/beta-catenin pathway in hepatocellular carcinoma. Journal of cellular and molecular medicine. 2018; 22: 315-29.

36. Zhang XL, Chen ML, Zhou SL. Fentanyl inhibits proliferation and invasion of colorectal cancer via beta-catenin. International journal of clinical and experimental pathology. 2015; 8: 227-35.

37. Xu T, Wu S, Yuan Y, Yan G, Xiao D. Knockdown of phosphodiesterase 4D inhibits nasopharyngeal carcinoma proliferation via the epidermal growth factor receptor signaling pathway. Oncology letters. 2014; 8: 2110-6.

38. Fang D, Hawke D, Zheng Y, Xia Y, Meisenhelder J, Nika H, et al. Phosphorylation of beta-catenin by AKT promotes beta-catenin transcriptional activity. The Journal of biological chemistry. 2007; 282: 11221-9.

39. Lu Z, Ghosh S, Wang Z, Hunter T. Downregulation of caveolin-1 function by EGF leads to the loss of E-cadherin, increased transcriptional activity of beta-catenin, and enhanced tumor cell invasion. Cancer cell. 2003; 4: 499-515. 